\title{
The simulation of cosmic rays in EUSO-Balloon: performances of the direction and energy reconstruction
}

\author{
F. Fenu*, M. Bertaina \\ Università degli studi di Torino, INFN Torino \\ E-mail: francesco.fenulgmail.com
}

A. Guzman, K. Shinozaki, T. Mernik, J. Bayer, A. Santangelo

Eberhard Karls Universität Tübingen

\section{N. Sakaki}

Osaka City University

\section{S. Bacholle, A. Jung, E. Parizot}

Universitè Paris Diderot, Paris 7

\section{for the JEM-EUSO Collaboration}

The EUSO-Balloon experiment is being developed as a pathfinder for the JEM-EUSO mission. In this framework we are developing a series of balloon flights, with a rescaled version of the JEM-EUSO detector, to be deployed between 30 and $40 \mathrm{~km}$ height. In view of a long duration flight, we estimate the feasibility of detecting real cosmic ray events. In this contribution we evaluate the energy and direction reconstruction performance for the EUSO-Balloon mission. We simulate several samples of EeV cosmic ray events, including the detector, and we apply the algorithms to reconstruct their energy and direction. We therefore show results on the energy and direction resolution and give an estimate of the fraction of good quality events with respect to the simulated events.

The 34th International Cosmic Ray Conference,

30 July- 6 August, 2015

The Hague, The Netherlands

\footnotetext{
* Speaker.
} 


\section{Introduction}

EUSO-Balloon [1] is a balloon-borne experiment developed as a pathfinder for the JEMEUSO mission [2]. This effort consists of a series of stratospheric balloon flights of various durations and at various altitudes. We plan to prove the technological readiness of the detector and to test the capability to cope, in space-like conditions, with a wide variety of atmospheric sources. We also aim at the first fluorescence cosmic ray detection from $40 \mathrm{~km}$ height. In August 2014 the JEM-EUSO collaboration successfully completed the first EUSO-Balloon flight from TimminsCanada. The balloon flew for 5 hours and was not equipped with a stand-alone trigger [4] in order to recognize the Cosmic Ray tracks since primary objective of the mission was to measure the background and to test the electronics stability in a real environment. For this reason the mission was not capable of detecting cosmic rays. In a future long duration flight equipped with a trigger electronics, instead, the detection of cosmic rays is possible. In another contribution to this conference [3] we prove the feasibility of the detection from the point of view of trigger exposure. Aim of the present contribution is, on the other hand, to prove the establishment of the reconstruction chain for the balloon and to test the quality of the reconstruction algorithms for a future balloon flight configuration. We will simulate samples of cosmic rays in fixed condition and test the reconstruction algorithms developed in the JEM-EUSO framework [5] [6]. We test the algorithms "as they are" and present preliminary results without any optimization on the specific Balloon signal morphology. We will give an estimation of the resolution of the direction and energy reconstruction. We will also give an estimation of the fraction of good quality events with respect to the simulated events.

\section{Cosmic ray balloon simulations}

In this section we show the typical simulated detector response of a cosmic ray event as seen by the EUSO-Balloon. This simulation is performed assuming a square lens of $1 \mathrm{~m}$ side at an altitude of $38 \mathrm{~km}$. The simulated photons are focussed by a system of three Fresnel lenses on a single Photo Detection Module (PDM) consisting of 36 Multi Anode Photomultipliers of 64 channels. The detection efficiency is set to 0.26 including both quantum and detection efficiency. This optics design has a 5.5 degrees $\times 5.5$ degrees field of view with a resolution of 0.2 degrees per pixel. In this simulation we include the first level trigger designed to reduce the fake trigger rate to a level compatible with electronics design constraints. In this way we can give a first estimate of a future long duration mission performances which will have a trigger system and will be able to autonomously trigger on cosmic ray events. Showers have been randomly injected on a surface of $20 \times 20 \mathrm{~km}^{2}$ (significantly larger than the $\sim 7.5 \times 7.5 \mathrm{~km}^{2}$ field of view) in order to avoid border effects. We simulated 1000 showers of fixed energy at $5 \times 10^{18}$ and $10^{19} \mathrm{eV}$ and zenith angle of 25 degrees. For the angular reconstruction we simulated showers from $10^{18}$ to $10^{19} \mathrm{eV}$ always at 25 degrees zenith angle. In all the cases we simulated all the azimuthal angles from 0 to 360 degrees.

In Fig. 1 we show the simulated response of EUSO-Balloon for a $5 \times 10^{18} \mathrm{eV}, 25$ degrees shower. In Fig. 2 we see instead the response for a 60 degree shower. As can be clearly seen at 25 degrees the shower is fully included in the field of view while the 60 degrees shower is just partially detected. On the other hand, in Fig. 3 we observe the signal for a 60 degrees $10^{20} \mathrm{eV}$ shower in 

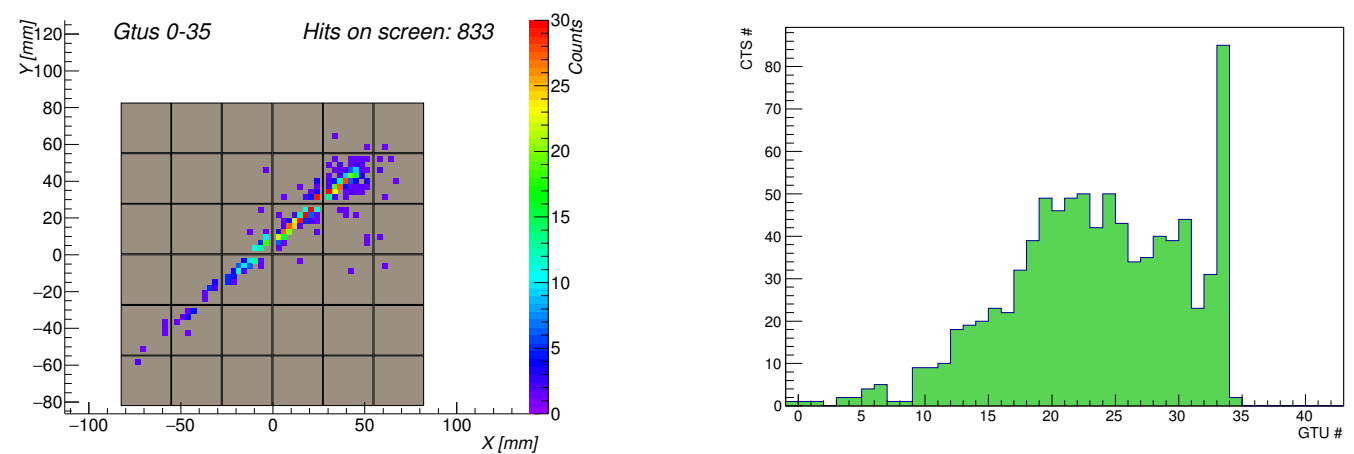

Figure 1: an example of a $5 \times 10^{18} \mathrm{eV} 25$ degrees shower image is shown on the left panel. Just simulated signal is drawn. On the right panel we see the simulated counts curve for the same shower. The final Cherenkov reflection mark can be seen at GTU 33.
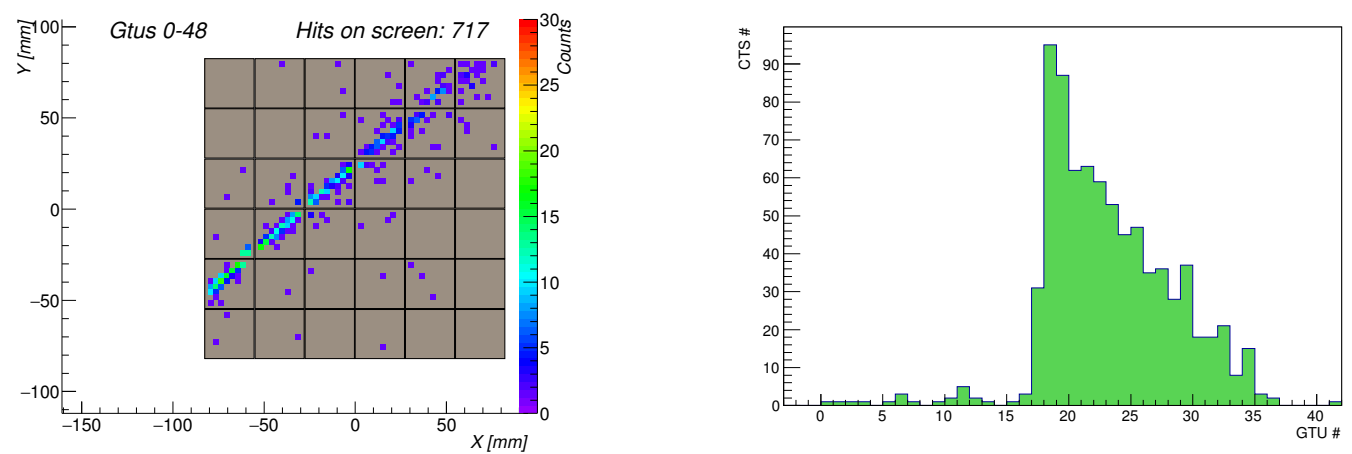

Figure 2: an example of a $5 \times 10^{18} \mathrm{eV} 60$ degrees shower image is shown on the left panel. Just simulated signal is drawn. The simulated counts curve for the same shower is shown on the right. We can clearly notice how the signal is cut in the first half of the propagation.

the JEM-EUSO case. We can notice how the entire signal is included in the PDM due to the larger size of the JEM-EUSO pixel on ground with respect to the Balloon.

Another feature which clearly distinguishes JEM-EUSO from EUSO-Balloon is the speed of the spot on the focal surface. In fact, the pixel size on ground is equal to $550 \mathrm{~m}$ on average for JEMEUSO against $200 \mathrm{~m}$ for EUSO-Balloon. This brings the EUSO-Balloon spot to be moving on the focal surface at a much higher speed than in the JEM-EUSO case. The algorithms for JEM-EUSO are however developed for a spot moving at most 1 pixel / GTU and therefore they are directly applicable just at low zenith angles. For this reason we temporarily limit our simulations in the low zenith angles interval. Some electronics and reconstruction optimization will be required in the future in order to take into account such differences in the signal morphology. However we prove the feasibility of the reconstruction of the triggered event also in the EUSO-Balloon configuration.

\section{The reconstruction algorithm}

For a more detailed explanation on the reconstruction procedure we refer to [7] [8] [9] [10]. In short, we apply a chain of algorithms to identify the signal in the triggered data, to reconstruct 

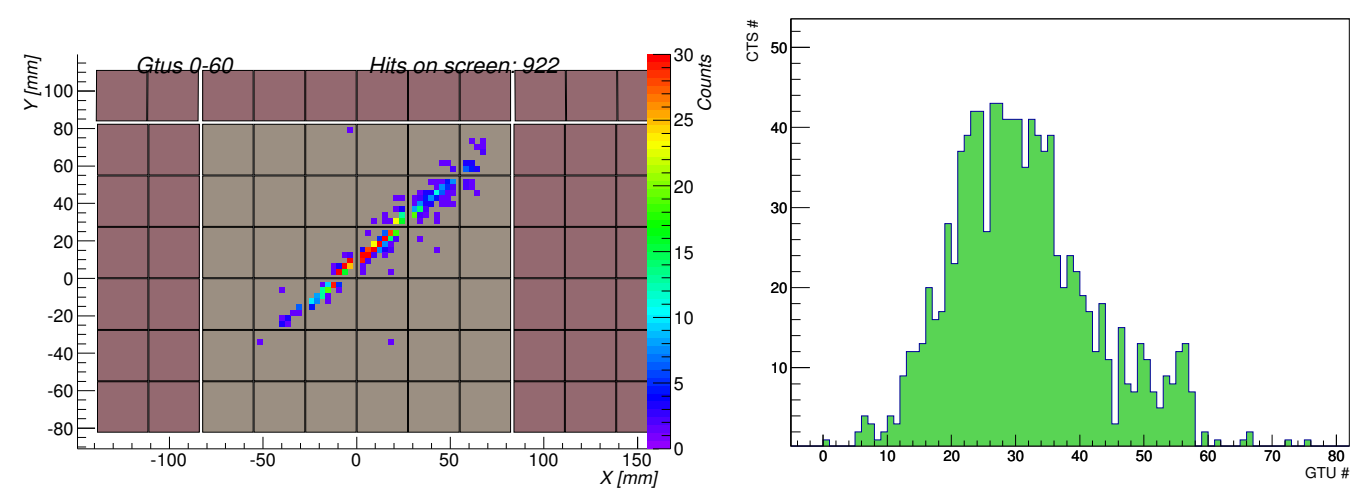

Figure 3: we show here the signal for a $10^{20} \mathrm{eV}, 60$ degrees JEM-EUSO shower. It can be easily seen how the entire track is included in a single PDM. The signal intensity is of the similar amplitude as a $5 \times 10^{18} \mathrm{eV}$ event for the balloon. Again on the left we see the image of the signal while on the right we see the signal curve.

the direction, the energy and $\mathrm{X}_{\max }$ of the shower. Several pattern recognition algorithms have been developed to identify the moving spot on the focal surface. Similarly to the trigger all of them aim to identify concentrations of signal moving coherently on the focal surface. The direction will be reconstructed by applying a fit on the direction and on the timing of the spot. Finally, a procedure to reconstruct the shower profile for the energy and $X_{\max }$ retrieval is also applied. The energy reconstruction gets the reconstructed signal pattern as input to correct all the inefficiencies of the detector, the atmospheric absorption and the fluorescence yield to eventually retrieve the shower profile. A fit will be then done on the profile and the obtained parameters are the energy and $\mathrm{X}_{\max }$. We show in this publication just some example that proves the establishment of the reconstruction chain and the feasibility of the event reconstruction from Balloon.

In Fig. 4 we can see an example of reconstructed shower profile. We can see here the real (black line) and reconstructed profile (points). The GIL ${ }^{1}$ fit on the selected points marked in red is represented as a continuous red line. As can be seen, the reconstructed profile is slightly overestimated (as in the case of JEM-EUSO [5]) due to the lack of backscattered Cherenkov correction. The profile is cut in the second half due to the impact with ground. The resulting Cherenkov reflection feature is also visible in the reconstructed profile. The shower of energy $10^{19} \mathrm{eV}$ and 25 degrees has been reconstructed as $1.1 \times 10^{19} \mathrm{eV}$. The $\chi^{2} /$ DOF is of 0.97 and the number of degrees of freedom of the fit is 12 .

In Figs. 5 and 6 we can see samples of reconstructed events in fixed condition. We report here just the events with a fit of at least 4 degrees of freedom and a $\chi^{2} /$ DOF of at most 3 . The zenith angle is of 25 degrees and the energies are $10^{19}$ and $5 \times 10^{18} \mathrm{eV}$. We calculated the parameter

$$
\Delta=\frac{E_{\text {reco }}-E_{\text {real }}}{E_{\text {real }}}
$$

for each event and we plotted it for a distribution of events. As can be seen a gaussian fit has been performed and is represented as red continuous line. The sigma of this distribution is 0.19 and 0.24 for the two cases and gives us an estimate for the energy resolution for the given set of true parameters.

\footnotetext{
${ }^{1}$ Gaisser Ilina Linsley, a parameterization to model the longitudinal profile of showers. See [5] for details.
} 


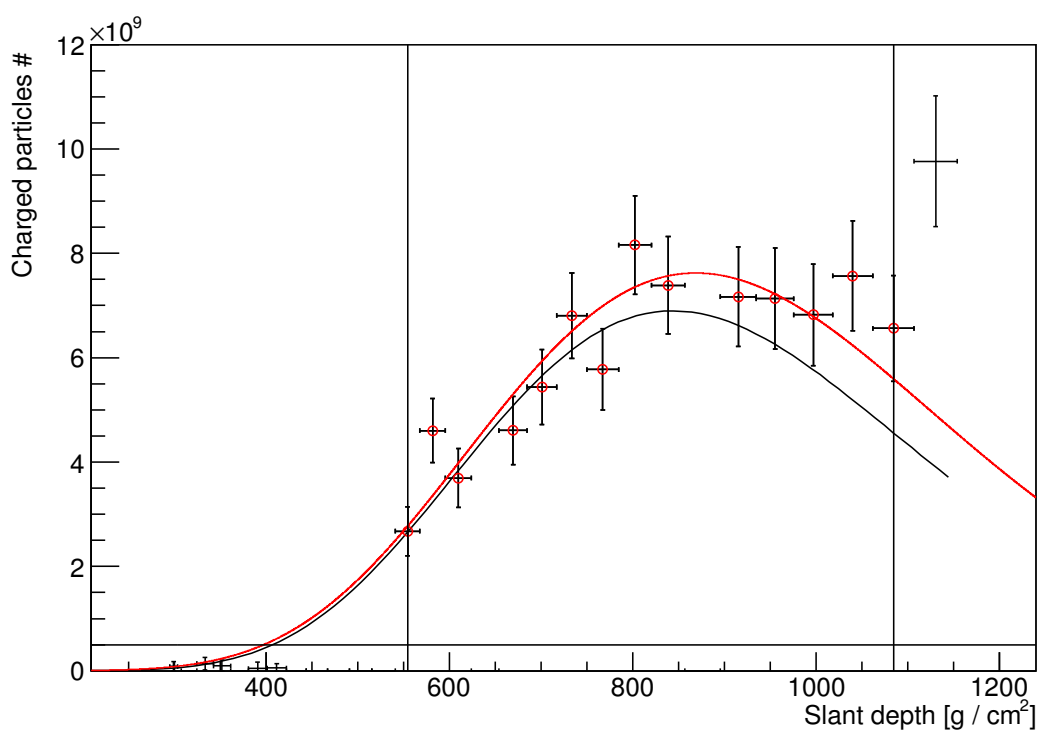

Figure 4: we show here an example of a reconstructed Balloon event. As continuous black line we see the simulated shower profile or the number of charged particles in the shower at each development stage. As black point we observe the reconstructed profile as obtained by the algorithms. We see marked in red all the points which are accepted as fit data and as continuous red line the GIL fit applied to the points. We clearly see how the automatic fit correctly excluded the Cherenkov mark visible at the end of the curve. The fitting range and the minimal threshold automatically chosen by the algorithms are also shown as black straight lines.

The high quality fraction is defined as following

$$
E f f_{\text {norm }}=\frac{N_{\text {reco }}}{N_{\text {simu }}} \times \frac{A_{\text {inj }}}{A_{\text {fov }}}
$$

whereas $\mathrm{N}_{\text {reco }}$ and $\mathrm{N}_{\text {simu }}$ are respectively the number of good quality events and the number of simulated events, $\mathrm{A}_{i n j}$ is the area of injection and $\mathrm{A}_{f o v}$ the area of the field of view. We simulated every time 1000 events over a surface of $400 \mathrm{~km}^{2}$ with a field of view of $51 \mathrm{~km}^{2}$. We can therefore calculate the efficiency for the two plots shown above where we see 100 and 90 events respectively. The efficiency turns out therefore to be 78 and $70 \%$ for $10^{19}$ and $5 \times 10^{18} \mathrm{eV}$.

To conclude we show in Fig. 7 the angular reconstruction obtained for events of energies between $10^{18}$ and $10^{19} \mathrm{eV}$ and 25 degrees zenith angle. We therefore calculated the angle $\gamma$ by the relation

$$
\gamma=\arccos \left(\hat{\Omega}_{\text {simu }} \cdot \hat{\Omega}_{\text {reco }}\right)
$$

whereas $\hat{\Omega}_{\text {simu }}$ and $\hat{\Omega}_{\text {reco }}$ are the unit vectors defining the direction of the simulated and reconstructed shower. We calculated the parameter

$$
\Gamma_{\text {int }}\left(\gamma^{*}\right)=\int_{0}^{\gamma^{*}} \frac{n_{\gamma}}{N_{T O T}} d \gamma
$$

which represents the integration of the renormalized $\gamma$ distribution density $\frac{n_{\gamma}}{N_{T O T}}$ from 0 to $\gamma^{*} . n_{\gamma}$ represents the number of events falling in a specific $\gamma$ bin and $\mathrm{N}_{T O T}$ the total number of reconstructed events. The parameter $\Gamma_{\text {int }}$ is used to find the so called $\gamma_{68 \%}$ namely the interval within 


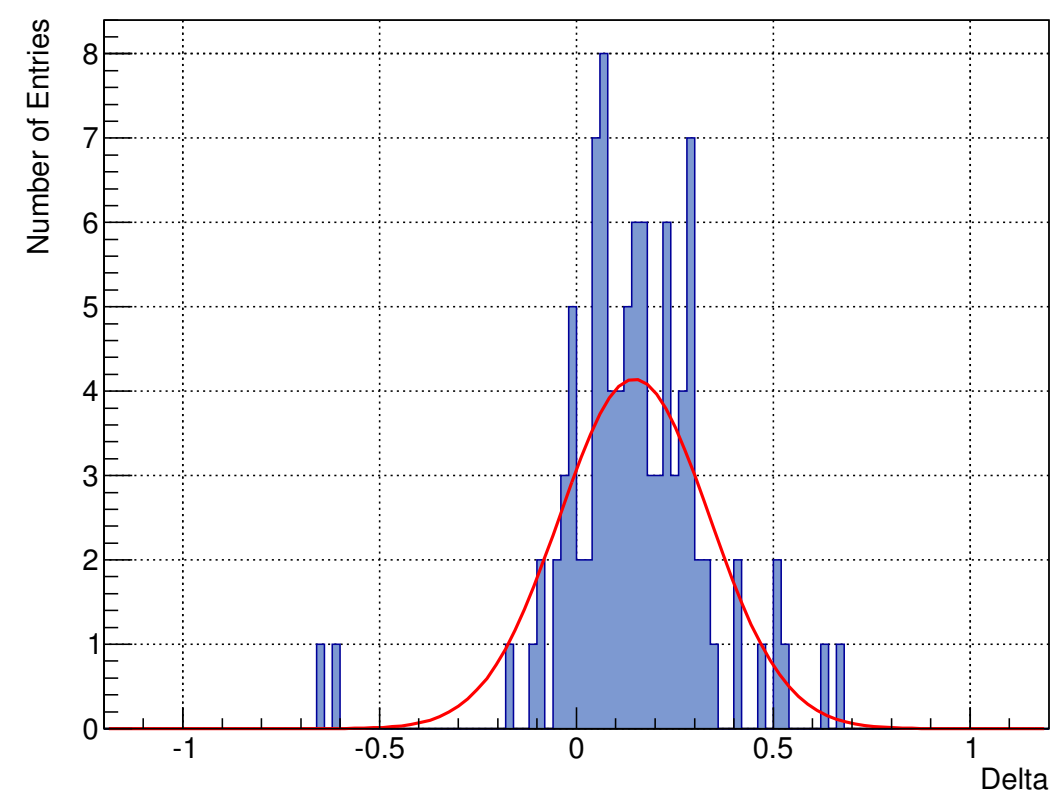

Figure 5: the $\Delta$ reconstruction parameter for $10^{19} \mathrm{eV}$ and 25 degrees. We fitted the distribution of such parameter over a sample of events with a gaussian function. The sigma of the distribution is of 0.19 . We can see a clear overestimation of the energy due to the lack of the backscattered Cherenkov correction.

which the $68 \%$ of the events is falling. We defined this parameter as angular resolution. According to this definition the angular resolution in such condition is therefore around 5 degrees.

\section{Conclusions}

In this contribution we proved the establishment of the reconstruction chain for the EUSOBalloon experiment. We proved that the software is set up and gave a minimal set of conditions where the reconstruction was tested. We started to identify the general tendencies and criticalities of the reconstruction. The signal spot is first of all much faster on the focal surface than it was for JEM-EUSO. As long as we apply the algorithms without optimization we are forced to use them just for low zenith angles. In this range we proved however that the algorithms work in a high fraction of cases with a resolution of the order of $20-25 \%$ above $5 \times 10^{18} \mathrm{eV}$ for the energy and with 5 degrees for the angle. We plan therefore to optimize the trigger and the reconstruction algorithms for the EUSO-Balloon detector to take into account the different morphology of the signal with respect to JEM-EUSO. Such results indicate however that a good fraction of the events triggered in a long duration flight can be reconstructed with good resolution. The EUSO-Balloon is therefore in good position to meet its main mission objective namely the detection and reconstruction of cosmic rays from near space in order to prove the feasibility of the JEM-EUSO detection concept.

Acknowledgment: This work was partially funded by the Italian Ministry of Foreign Affairs, General Direction for the Cultural Promotion and Cooperation. We thank the original ESAF developers for their work. 


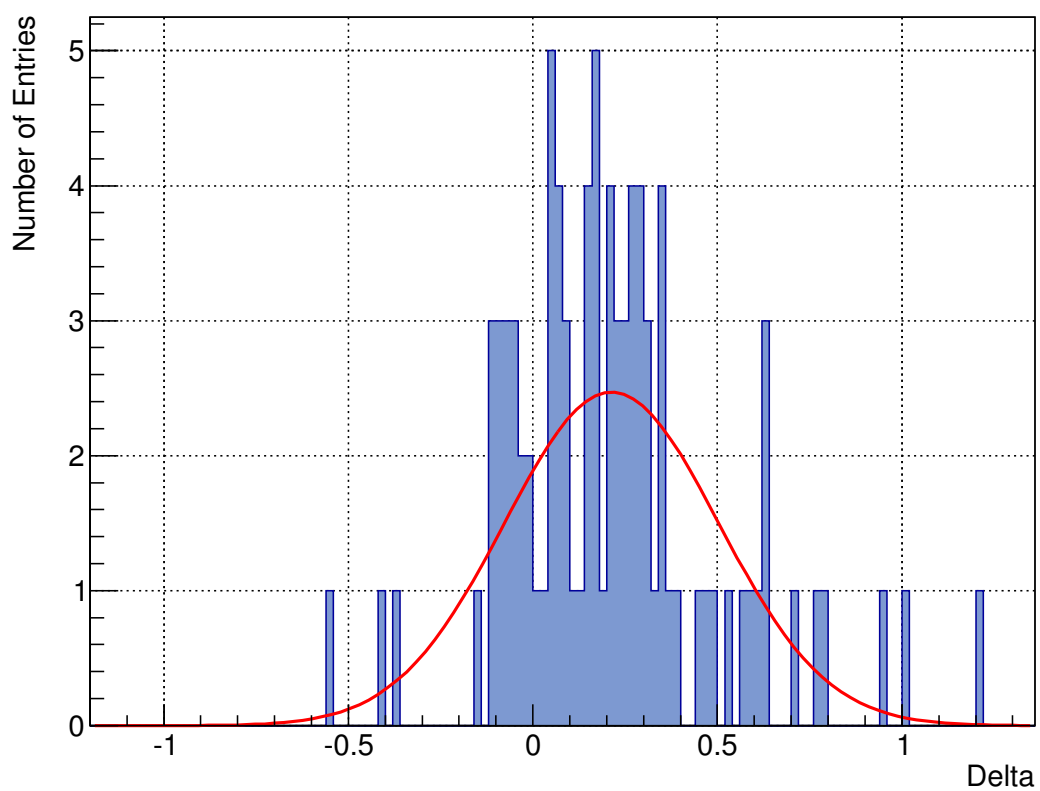

Figure 6: the $\Delta$ reconstruction parameter for $5 \times 10^{18} \mathrm{eV}$ and 25 degrees. We fitted the distribution of such parameter over a sample of events with a gaussian function. The sigma of the distribution is of 0.24 . We can see a clear overestimation of the energy due to the lack of the backscattered Cherenkov correction.

\section{References}

[1] "EUSO-Balloon: A pathfinder mission for the JEM-EUSO experiment", G. Osteria et al., Nuclear Instruments and Methods in Physics Research Sect. A, Volume 732, 2013, Pag. 320-324

[2] "The JEM-EUSO mission", T. Ebisuzaki et al., for the JEM-EUSO Collaboration, Advances in Space Research Vol. 53, Issue 10, 2014, Pages 1499-1505

[3] "EUSO-Balloon trigger efficiency in preparation of a long duration flight", S. Bacholle et al., This conference contribution

[4] "Test of JEM-EUSO $1^{\text {st }}$ level trigger using the EUSO-Balloon data", M. Bertaina et al., This conference contribution

[5] "Performances of JEM-EUSO: energy and $\mathrm{X}_{\max }$ reconstruction", F. Fenu et al., accepted for publication, Experimental Astronomy

[6] "The JEM-EUSO energy and $X_{\max }$ reconstruction performances", F. Fenu et al., This conference contribution

[7] "The Peak and Window Searching Technique for the EUSO Simulation and Analysis Framework: Impact on the Angular Reconstruction of EAS", A. Guzman et al., 2013, Journal of physics: conference series, vol. 409 conf. 1

[8] "Performances of JEM-EUSO: angular reconstruction", Bitkemerova et al., 2014, Experimental Astronomy 


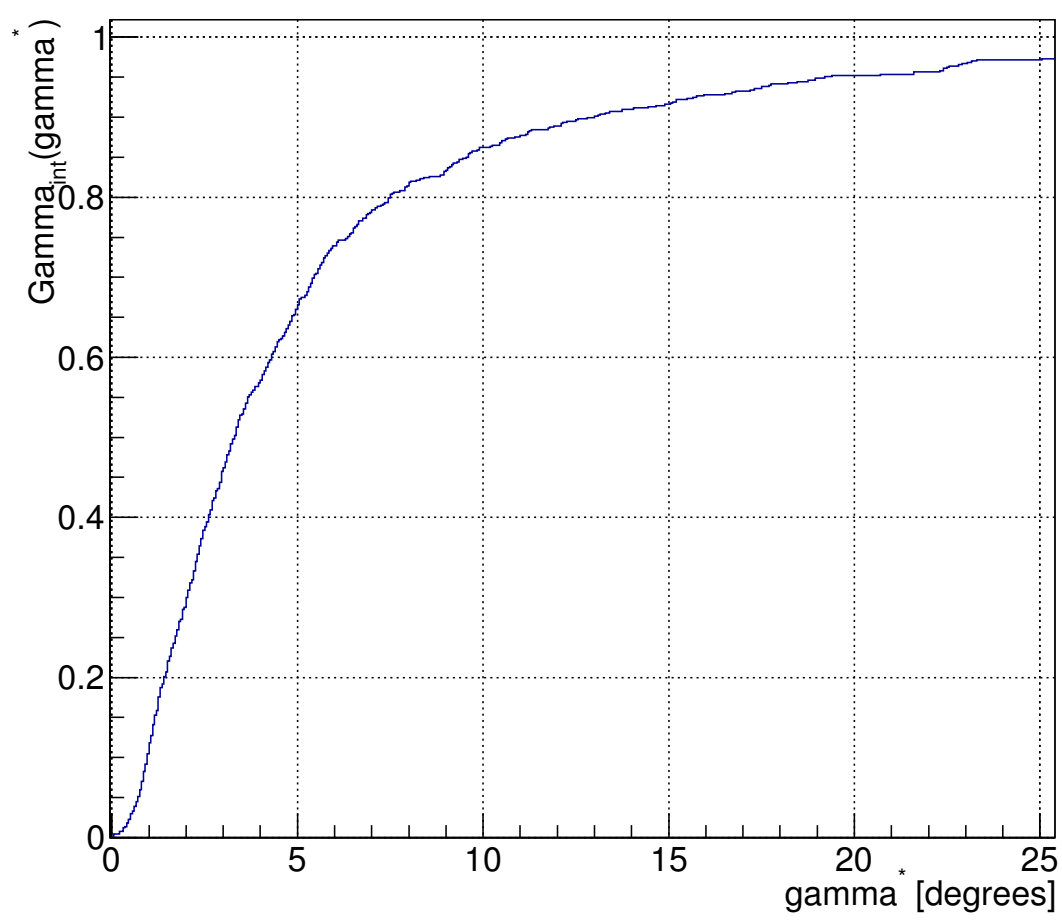

Figure 7: the $\Gamma_{\text {int }}\left(\gamma^{*}\right)$ parameter to define the angular resolution for a distribution of events having zenith angle of 25 degrees and energies from $10^{18}$ to $10^{19} \mathrm{eV}$. As can be seen the $68 \%$ value is reached around 5 degrees.

[9] "A simulation study of the JEM-EUSO mission for the detection of ultra-high energy Cosmic Rays", F. Fenu, 2013, PhD Thesis, Tübingen

[10] "The Expected Angular Resolution of the JEM-EUSO Mission", T. Mernik, 2014, PhD Thesis, Tübingen 